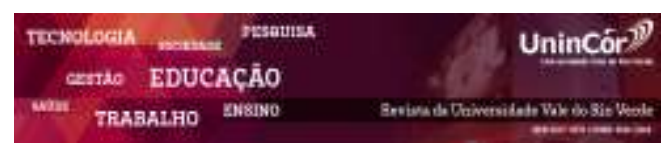

Revista da Universidade Vale do Rio Verde ISSN: 1517-0276 / EISSN: 2236-5362 Vol. $16 \mid$ n. 2| Ano 2018

Alessandro Becker Faculdade Meridional (IMED) alessandrobecker@gmail.com

Eliana Andréa Severo Universidade Potiguar (UnP) elianasevero2@hotmail.com

Julio Cesar Ferro de Guimarães Universidade Potiguar (UnP) juliocfguimaraes@yahoo.com.br

\section{UMA REVISÃO SISTEMÁTICA DA LITERATURA SOBRE INOVAÇÃO E SUA RELAÇÃO COM O AGRONEGÓCIO}

\section{RESUMO}

A inovação possui papel importante no mercado e precisa estar presente no campo do agronegócio pois com a atual dinâmica do mercado tem-se utilizado práticas que envolvam a tecnologia no campo em produtos, processos, marketing e organizacional. $\mathrm{O}$ objetivo desse estudo foi identificar se os artigos mais citados e recentes quanto a palavra-chave inovação tem relação com o tema do agronegócio. A metodologia utilizada trata-se de uma abordagem qualitativa de caráter exploratório por meio de uma revisão sistemática na Base de dados Scopus. Para a análise de dados utilizou-se a técnica de análise de conteúdo. Os resultados encontrados demonstram que os artigos mais citados e recentes de inovação na base de dados Scopus não abordam diretamente o agronegócio, mas por outro lado pode ser aproveitado para aprofundamento de estudos e práticas de forma indireta. Coerentemente, é fundamental que o setor agroindustrial desenvolva inovações que favoreçam o sistema de escoamento e armazenamento das safras, reduzindo custos logísticos, tanto para operadores, quanto usuários, possibilitando melhoria no processo produtivo e performance organizacional.

Palavras-chave: Inovação. Relação. Agronegócio. Base de dados Scopus. Revisão sistemática.

\section{A SYSTEMATIC REVIEW OF THE LITERATURE ON INNOVATION AND ITS RELATIONSHIP WITH AGRIBUSINESS}


Keywords: Innovation. Relationship. Agribusiness. Scopus database. Systematic review.

Recebido em: 20/02/2018 - Aprovado em: 25/07/2018 - Disponibilizado em: 15/12/2018

\section{INTRODUÇÂO}

Inovação é um tema de diversas pesquisas, pois visa a performance e competitividade das organizações. Vem sendo abordado desde a década de 30 por Joseph Alois Schumpeter, na década de 90 pelo Manual do OSLO (2005), e recentemente por Park e Koh (2017), os quais destacam que a inovação é vista como o motor do desenvolvimento econômico e sustentabilidade das organizações. Para Severo, Guimarães e Dorion (2017), a inovação pode se tornar fundamental para a promoção da competitividade das organizações no contexto regional e também global, primando para a sustentabilidade ambiental.

Guimarães (2013) corrobora nesse sentido, pois considera a inovação importante para o desenvolvimento socioeconômico de uma região, visto que reside na capacidade de transformação econômica, mas ressalta a necessidade da empresa adotar a inovação como parte de sua estratégia.

Nesse cenário, o agronegócio é umas das mais importantes fontes geradoras de riqueza do Brasil, pois representa cerca de $30 \%$ do Produto Interno Bruto (PIB) e coloca o país entre as nações mais competitivas do mundo na produção de commodities agroindustriais (JANK; NASSAR; TACHINARDI; 2004). Conforme Sabadin, Severo e Guimarães o agronegócio é um dos setores que gera desenvolvimento, empregos, PIB para o país, estimula a agricultura familiar e as exportações.

Atualmente, o agronegócio tem assumido importante papel na economia brasileira, pois as suas empresas têm usado de diferentes formatos de gestão para incrementar a produção e, principalmente, para manterem-se lucrativas (ASAI et al, 2017). Ressalta-se que em 2015 mesmo diante da economia desfavorável e da redução dos preços das principais commodities, o agronegócio brasileiro teve uma safra recorde, fechando o ano com a balança comercial superavitária. Consoante isso, Santos et al. (2016) destacam que o agronegócio brasileiro contribuiu significativamente para o equilíbrio da balança comercial do país. De acordo com dados do Centro de Estudos Avançados em Economia Aplicada (CEPEA, 2016), o PIB do agronegócio cresceu 0,39\% em comparação ao ano de 2014.

Para Batalha (1995), a tecnologia desempenha papel relevante como fator explicativo das estruturas industriais e do comportamento competitivo das agroindústrias. O autor considera importante integrar o estudo das inovações tecnológicas ao conjunto de ações de reflexão estratégica das empresas. King et al. (2010), consideram que o setor agrícola é cada vez mais uma fonte de matéria-prima para setores além do sistema tradicional de alimentos e fibras, ocasionando desafios competitivos e trazendo profundas implicações para a estrutura 
e operações das cadeias de suprimentos na indústria.

Nesse contexto, o agronegócio necessita avançar para a sustentabilidade, incorporando outras dimensões que perpassam a tradicional orientação para a dimensão econômicofinanceira (DIAS; PEDROZO; ANICET, 2011). Coerentemente, a busca pela inovação pretende garantir a posição e/ou ampliação da participação das empresas no mercado (SEVERO, 2013). Contudo, perante a importância da inovação no âmbito organizacional, surge um elo entre inovação e agronegócios, bem como necessita-se de pesquisas que avaliem a inovação no agronegócio.

Perante o exposto, esse estudo busca analisar as pesquisas sobre inovação e sua relação com o agronegócio. Além dessa introdução, o artigo está estruturado em material e métodos, resultados e discussões e considerações finais.

\section{MATERIAL E MÉTODOS}

A metodologia utilizada nesse estudo trata-se de uma pesquisa qualitativa de caráter exploratório (GIBBS, 2009; CRESWELL, 2007). Consoante isso, foi operacionalizada por meio de uma revisão bibliográfica (WOLFSWINKEL; FURTMUELLER; WILDEROM; 2013) e sistemática na base de dados Scopus.

Com o intuito de realizar a pesquisa sistemática sobre o tema (inovação), primeiramente, foram verificadas as bases de dados, na qual a pesquisa seria realizada. Após um levantamento das possibilidades, optou-se por utilizar a base Scopus, por se tratar de umas das principais bases de dados de resumos e citações de literatura científica disponível. O processo de pesquisa foi realizado entre os dias 11 de janeiro e 14 de fevereiro de 2017.

A pesquisa na base de dados Scopus foi realizada por meio de consulta dos artigos a partir da pesquisa da palavra-chave innovation. Após a identificação dos artigos, foram selecionados os 10 artigos mais citados e os 10 artigos mais recentes.

A Figura 1 apresenta o delineamento do processo da metodologia de pesquisa utilizado. Contudo, utilizaram-se também demais fontes, tais como livro, outros artigos, dissertação e tese para a consolidação das discussões e considerações finais.

Para a análise e interpretação de dados utilizou-se a técnica de análise de conteúdo (BARDIN, 2002), a qual configura-se como um conjunto de técnicas de análise das informações, que faz uso de procedimentos sistemáticos e objetivos de descrição do conteúdo das mensagens.

Figura 1 - Sistemática da pesquisa

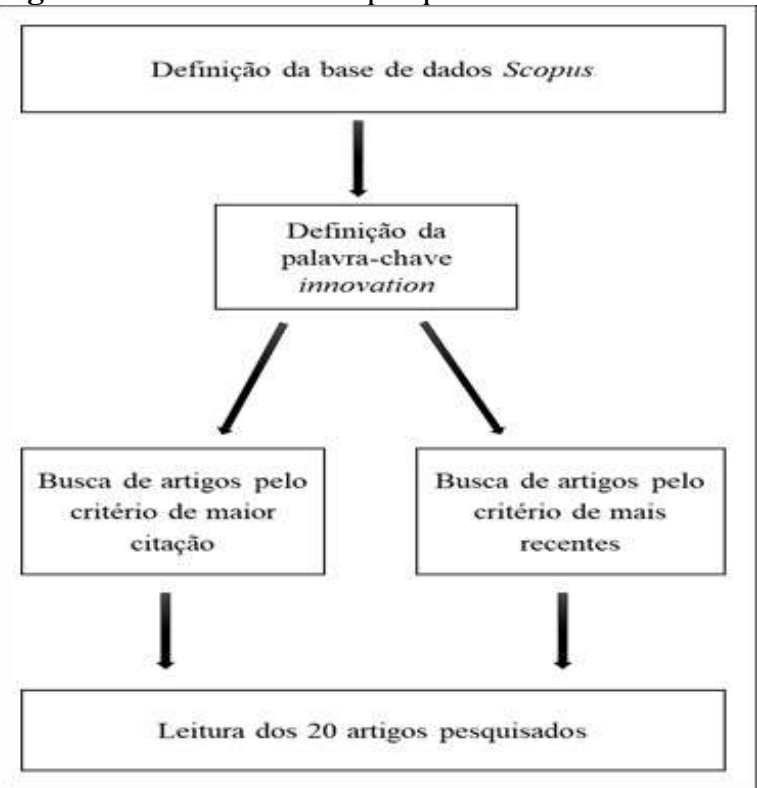

Fonte: Elaborado pelos autores (2017). 


\section{RESULTADOS E DISCUSSÃO}

No levantamento de informações realizado foram identificados 130.987
Quadros 1 e 2 apresentam a sistematização dos artigos mais citados e recentes com relação a palavra-chave pesquisada, bem como o nome dos autores e periódico, e o assunto discutido.

documentos para a palavra-chave innovation. Os

Quadro 1 - Artigos mais citados com a palavra innovation

\begin{tabular}{|c|c|c|c|c|c|}
\hline $\begin{array}{l}\text { Número de } \\
\text { citações }\end{array}$ & Título do artigo & Autor (es) & Ano & Nome do periódico & Assunto \\
\hline 9055 & $\begin{array}{l}\text { Dynamic capabilities } \\
\text { and strategic manage- } \\
\text { ment }\end{array}$ & $\begin{array}{l}\text { Teece, Pisa- } \\
\text { no e Shuen }\end{array}$ & 1997 & $\begin{array}{l}\text { Strategic Manage- } \\
\text { ment Journal }\end{array}$ & $\begin{array}{l}\text { Capacidades dinâmicas em } \\
\text { ambientes de rápida mudan- } \\
\text { ça. }\end{array}$ \\
\hline 7702 & $\begin{array}{l}\text { User acceptance of } \\
\text { information technology: } \\
\text { Toward a unified view }\end{array}$ & $\begin{array}{l}\text { Venkatesh et } \\
\text { al. }\end{array}$ & 2003 & $\begin{array}{l}\text { MIS Quarterly: } \\
\text { Management Infor- } \\
\text { mation Systems }\end{array}$ & $\begin{array}{l}\text { Aceitação da tecnologia da } \\
\text { informação e modelos pro- } \\
\text { eminentes. }\end{array}$ \\
\hline 4662 & $\begin{array}{l}\text { The balanced score- } \\
\text { card--measures that } \\
\text { drive performance }\end{array}$ & $\begin{array}{l}\text { Kaplan e } \\
\text { Norton }\end{array}$ & 1992 & $\begin{array}{l}\text { Harvard Business } \\
\text { Review }\end{array}$ & $\begin{array}{l}\text { Medição de desempenho } \\
\text { organizacional. }\end{array}$ \\
\hline 3252 & $\begin{array}{l}\text { Development of an in- } \\
\text { strument to measure the } \\
\text { perceptions of adopting } \\
\text { an information technol- } \\
\text { ogy innovation }\end{array}$ & $\begin{array}{l}\text { Moore e } \\
\text { Benbasat }\end{array}$ & 1991 & $\begin{array}{l}\text { Information Systems } \\
\text { Research }\end{array}$ & $\begin{array}{l}\text { Desenvolvimento de instru- } \\
\text { mento para medir percep- } \\
\text { ções de indivíduos sobre } \\
\text { adotar uma inovação em } \\
\text { tecnologia da informação. }\end{array}$ \\
\hline 2948 & $\begin{array}{l}\text { Understanding informa- } \\
\text { tion technology usage: } \\
\text { A test of competing } \\
\text { models }\end{array}$ & $\begin{array}{l}\text { Taylor e } \\
\text { Todd }\end{array}$ & 1995 & $\begin{array}{l}\text { Information Systems } \\
\text { Research }\end{array}$ & $\begin{array}{l}\text { Comparação entre modelo } \\
\text { de aceitação da tecnologia e } \\
\text { variações da teoria do com- } \\
\text { portamento planejado quan- } \\
\text { to ao uso de tecnologia da } \\
\text { informação. }\end{array}$ \\
\hline 2548 & $\begin{array}{l}\text { Core capabilities and } \\
\text { core rigidities: A para- } \\
\text { dox in managing new } \\
\text { product development }\end{array}$ & $\begin{array}{l}\text { Leonard- } \\
\text { Barton }\end{array}$ & 1992 & $\begin{array}{l}\text { Strategic Manage- } \\
\text { ment Journal }\end{array}$ & $\begin{array}{l}\text { Natureza das capacidades } \\
\text { básicas de uma empresa } \\
\text { com foco na sua interação } \\
\text { com novos projetos de de- } \\
\text { senvolvimento de produtos e } \\
\text { processos. }\end{array}$ \\
\hline 2272 & $\begin{array}{l}\text { The price of innovation: } \\
\text { New estimates of drug } \\
\text { development costs }\end{array}$ & $\begin{array}{l}\text { DiMasi, } \\
\text { Hansen e } \\
\text { Grabowski } \\
\end{array}$ & 2003 & $\begin{array}{l}\text { Journal of Health } \\
\text { Economics }\end{array}$ & $\begin{array}{l}\text { Custos de pesquisa e desen- } \\
\text { volvimento de novas drogas } \\
\text { farmacêuticas. }\end{array}$ \\
\hline 2172 & $\begin{array}{l}\text { Diffusion of innovations } \\
\text { in service organiza- } \\
\text { tions: Systematic review } \\
\text { and recommendations }\end{array}$ & $\begin{array}{l}\text { Greenhalgh } \\
\text { et al. }\end{array}$ & 2004 & Milbank Quarterly & $\begin{array}{l}\text { Disseminação e manutenção } \\
\text { de inovações na prestação } \\
\text { de serviços de saúde. }\end{array}$ \\
\hline 2075 & $\begin{array}{l}\text { What's your strategy for } \\
\text { managing knowledge? }\end{array}$ & $\begin{array}{l}\text { Hansen, } \\
\text { Nohria e } \\
\text { Tierney }\end{array}$ & 1999 & $\begin{array}{l}\text { Harvard business } \\
\text { review }\end{array}$ & $\begin{array}{l}\text { Práticas de gestão do co- } \\
\text { nhecimento em empresas de } \\
\text { consultoria de gestão, pres- } \\
\text { tadores de cuidados de saú- } \\
\text { de e fabricantes de compu- } \\
\text { tadores. }\end{array}$ \\
\hline 1946 & $\begin{array}{l}\text { Explicating dynamic } \\
\text { capabilities: The nature } \\
\text { and microfoundations } \\
\text { of (sustainable) enter- } \\
\text { prise performance }\end{array}$ & Teece & 2007 & $\begin{array}{l}\text { Strategic Manage- } \\
\text { ment Journal }\end{array}$ & $\begin{array}{l}\text { Capacidades necessárias } \\
\text { para sustentar o desempenho } \\
\text { empresarial superior em } \\
\text { uma economia aberta com } \\
\text { inovação rápida e fontes } \\
\text { globalmente dispersas de } \\
\text { invenção, inovação e capa- } \\
\text { cidade de fabricação. }\end{array}$ \\
\hline
\end{tabular}

Fonte: Elaborado pelos autores (2017). 
Quadro 2 - Artigos mais recentes com a palavra innovation

\begin{tabular}{|c|c|c|c|c|}
\hline Título do artigo & Autor (es) & Ano & Nome do periódico & Assunto \\
\hline $\begin{array}{l}\text { Graphic design and social } \\
\text { networks: Methodological } \\
\text { proposal supported by the } \\
\text { open innovation and co- } \\
\text { creation }\end{array}$ & Hernandez & 2017 & $\begin{array}{l}\text { Advances in } \\
\text { Intelligent Systems } \\
\text { and Computing }\end{array}$ & $\begin{array}{l}\text { Metodologia para o processo de } \\
\text { design gráfico com participação } \\
\text { pública na tomada de decisões } \\
\text { através de redes sociais. }\end{array}$ \\
\hline $\begin{array}{l}\text { Open data: Quality over } \\
\text { quantity }\end{array}$ & $\begin{array}{l}\text { Sadiq e } \\
\text { Indulska }\end{array}$ & 2017 & $\begin{array}{l}\text { International Journal } \\
\text { of Information } \\
\text { Management }\end{array}$ & $\begin{array}{l}\text { Desafios em lidar com a } \\
\text { qualidade dos dados de conjuntos } \\
\text { de dados abertos. }\end{array}$ \\
\hline $\begin{array}{l}\text { Understanding determinants } \\
\text { and barriers of mobile } \\
\text { shopping adoption using } \\
\text { behavioral reasoning theory }\end{array}$ & Gupta e Arora & 2017 & $\begin{array}{l}\text { Journal of Retailing } \\
\text { and Consumer } \\
\text { Services }\end{array}$ & $\begin{array}{l}\text { Adoção de compras móveis } \\
\text { usando uma nova abordagem da } \\
\text { teoria do raciocínio } \\
\text { comportamental. }\end{array}$ \\
\hline $\begin{array}{l}\text { The effects of cultural } \\
\text { dimension on ICT } \\
\text { innovation: Empirical } \\
\text { analysis of mobile phone } \\
\text { services }\end{array}$ & $\begin{array}{l}\text { Bankole e } \\
\text { Bankole }\end{array}$ & 2017 & $\begin{array}{l}\text { Telematics and } \\
\text { Informatics }\end{array}$ & $\begin{array}{l}\text { Cultura como determinante } \\
\text { crucial das inovações. }\end{array}$ \\
\hline $\begin{array}{l}\text { The contingent effect of } \\
\text { analyst coverage: how does } \\
\text { analyst coverage affect } \\
\text { innovation and Tobin's } Q ?\end{array}$ & Jung & 2017 & $\begin{array}{l}\text { Asia-Pacific Journal } \\
\text { of Accounting and } \\
\text { Economics }\end{array}$ & $\begin{array}{l}\text { Relação entre analistas e } \\
\text { inovação. }\end{array}$ \\
\hline $\begin{array}{l}\text { On the estimation of missing } \\
\text { values in } A R(1) \text { model with } \\
\text { exponential innovations }\end{array}$ & $\begin{array}{l}\text { Saadatmand, } \\
\text { Nematollahi e } \\
\text { Sadooghi- } \\
\text { Alvandi } \\
\end{array}$ & 2017 & $\begin{array}{l}\text { Communications in } \\
\text { Statistics - Theory and } \\
\text { Methods }\end{array}$ & $\begin{array}{l}\text { Estimativa de valor faltante para } \\
\text { processo autoregressivo } \\
\text { estacionário com inovações } \\
\text { exponenciais. }\end{array}$ \\
\hline $\begin{array}{l}\text { Exploring the relationship } \\
\text { between perceived pace of } \\
\text { technology change and } \\
\text { adoption resistance to } \\
\text { convergence products }\end{array}$ & Park e Koh & 2017 & $\begin{array}{l}\text { Computers in Human } \\
\text { Behavior }\end{array}$ & $\begin{array}{l}\text { Relação entre comportamentos } \\
\text { dos consumidores com o ritmo da } \\
\text { inovação tecnológica. }\end{array}$ \\
\hline $\begin{array}{l}\text { eHealth adoption factors in } \\
\text { medical hospitals: A focus on } \\
\text { the Netherlands }\end{array}$ & $\begin{array}{l}\text { Faber, Van } \\
\text { Geenhuizen e } \\
\text { De Reuver }\end{array}$ & 2017 & $\begin{array}{l}\text { International Journal } \\
\text { of Medical Informatics }\end{array}$ & $\begin{array}{l}\text { Utilização efetiva de } \text { eHealth em } \\
\text { hospitais europeus. }\end{array}$ \\
\hline $\begin{array}{l}\text { Implementing artifacts: An } \\
\text { interactive frame analysis of } \\
\text { innovative educational } \\
\text { practices }\end{array}$ & $\begin{array}{l}\text { Vermeir, } \\
\text { Kelchtermans e } \\
\text { Marz }\end{array}$ & 2017 & $\begin{array}{l}\text { Teaching and Teacher } \\
\text { Education }\end{array}$ & Práticas educacionais inovadoras. \\
\hline $\begin{array}{l}\text { Feasibility Evaluation and } \\
\text { Optimization of a Smart } \\
\text { Manufacturing System Based } \\
\text { on } 3 D \text { Printing: A Review }\end{array}$ & Chen e Lin & 2017 & $\begin{array}{l}\text { International Journal } \\
\text { of Intelligent Systems }\end{array}$ & $\begin{array}{l}\text { Viabilidade do sistema de } \\
\text { fabricação inteligente baseado em } \\
\text { impressão 3D. }\end{array}$ \\
\hline
\end{tabular}

Fonte: Elaborado pelos autores (2017).

Conforme o Quadro 1, em relação à palavra inovação, os artigos mais citados foram publicados no período entre de 1991 e 2007. Os principais assuntos abordados nesses artigos tratam de inovação com o viés das capacidades dinâmicas em ambientes de rápidas mudanças, o papel de tecnologia da informação que está cada vez mais presente nos negócios, medição de desempenho organizacional, desenvolvimento de novos produtos e serviços, custos de pesquisa e desenvolvimento, manutenção de inovações e práticas de gestão do conhecimento nas organizações.

Nesse contexto, o Quadro 2, os artigos mais recentes para a mesma temática abordam design gráfico e redes sociais, desafios da qualidade de dados abertos, compras móveis e mobilidade, o papel crucial da cultura nas inovações, relacionamento entre analistas com 
inovação, relação entre comportamentos dos consumidores com o ritmo das inovações e práticas educacionais inovadoras.

As empresas precisam identificar novas oportunidades e organizá-las de forma eficaz e eficiente para aproveitá-las e isso pode ser mais importante para a geração de riqueza do que a estratégia em si (TEECE; PISANO; SCHUEN, 1997). Neste cenário, é crescente o uso de tecnologia da informação e a aceitação por parte do usuário vem sendo pesquisada. Venkatesh et al. (2003) desenvolveram um modelo para gestores avaliarem a probabilidade de sucesso das introduções de novas tecnologias. A comparação entre modelo de aceitação e variações do comportamento foi tema de estudo de Taylor e Pidd (1995). Também estão sendo desenvolvidos instrumentos para medir percepções de indivíduos sobre a adoção de inovação em tecnologia da informação (MOORE; BENBASAT, 1991).

A inovação é o instrumento específico do empreendedor e tanto um quanto outro são necessários para a sociedade e para a economia (DRUCKER, 1985). Nesse cenário, os gerentes de novos projetos de desenvolvimento de produtos e processos vêm enfrentando um paradoxo: como aproveitar as capacidades essenciais instaladas e lidar com a rigidez dos processos organizacionais, sendo que os projetos desempenham um papel importante nas estratégias emergentes, destacando a necessidade de mudança e liderando o caminho? (LEONARD-BARTON, 1992).

A inovação está presente em empresas de serviços e, para Greenhalgh et al. (2004), também estão sendo disseminadas e mantidas inovações em serviços, especificamente os relacionados à saúde. Já Di Masi, Hansen e Grabowski (2003) identificaram que os custos de pesquisa e desenvolvimento (P\&D) aumentaram a uma taxa anual de 7,4\% acima da inflação. Kaplan e Norton (1992) desenvolveram um modelo de medição que busca o equilíbrio entre medidas financeiras e operacionais e que permite visualizar a empresa de várias perspectivas simultaneamente.

Um outro viés que a inovação está recebendo a atenção é referente às práticas de gestão do conhecimento. Nessa direção, para Hansen, Nohria e Tierney (1999), a escolha da estratégia de gestão de conhecimento da empresa não é arbitrária e deve ser direcionada pela estratégia competitiva da empresa. Os autores alertam para o fato de que a gestão do conhecimento não deve ser isolada em um departamento funcional como recursos humanos (RH) ou tecnologia da informação (TI). Eles enfatizam que os benefícios são maiores, tanto para a empresa quanto para seus clientes.

As capacidades dinâmicas são necessárias para sustentar o desempenho empresarial superior em uma economia aberta com inovação rápida e fontes globalmente dispersas de invenção, inovação e capacidade de fabricação, bem como os dados abertos ou públicos. Isso contribui para desbloquear o potencial de inovação de empresas e governos (TEECE, 2007; SADIQ; INDUSKA, 2017).

Recentemente, a inovação aberta e a cocriação têm recebido ênfase dos pesquisadores. Nesse cenário, para Hernández (2017), o processo de design gráfico - ferramenta de comunicação que trabalha com sinais visuais, com a participação pública na tomada de decisões por meio de redes sociais - é uma alternativa. A tecnologia vem transformando e inovando o 
mundo dos negócios e, na visão de Gupta e Arora (2017), a utilização de plataformas móveis para compras vem influenciando o raciocínio comportamental dos consumidores.

Em pesquisa realizada por Bankole e Bankole (2017) sobre a influência da cultura na inovação, os resultados indicaram que a cultura é um conceito apropriado para descrever de que modo a inovação nas tecnologias de informação e comunicação pode ser influenciada pelo comportamento humano e, por isso, essa tecnologia é um determinante crucial das inovações. Os autores complementam que a inovação é uma das principais forças para o desenvolvimento socioeconômico e, assim, tornase o elemento chave para estimular o crescimento da economia de uma nação.

Os comportamentos dos consumidores estão relacionados com o ritmo da inovação tecnológica. Na visão de Park e Koh (2017), a expectativa de que algo mais novo, melhor e mais barato esteja disponível no momento inicial de lançamento irá influenciar fortemente a resistência dos consumidores à adoção dessa tecnologia inicial e mais cara e, nesse contexto, poderá prevalecer sua vontade de adiar a compra, de modo a esperar mais mudanças e melhorias.

Em estudo sobre a relação entre a equipe de analistas e o processo de inovação, Jung (2017) identificou que embora seja caro para as empresas se engajar nas atividades associadas ao aprimoramento do relacionamento com os analistas, há benefícios em termo de retorno sobre o investimento para compensar esses custos. Conforme o autor, inicialmente existem custos e o retorno é baixo, mas, depois, a empresa obtém resultados, ou seja, no médio e longo prazo, tornase vantajoso.

\section{CONSIDERAÇÕES FINAIS}

Com esse estudo foi possível identificar que o tema do agronegócio não tem sido foco de pesquisa de forma direta por meio das publicações mais recentes e citadas do tema inovação na base de dados Scopus. É importante considerar que indiretamente, várias pesquisas podem ser relacionadas com o agronegócio e com isso contribuir para a evolução do tema.

Cabe ressaltar que, se por um lado não foi identificada relação direta, por outro, apresenta-se a oportunidade de estudos que façam essa relação, pois o agronegócio é importante para a economia local e mundial, seja por movimentação de recurso financeiros, quanto pela geração de empregos. Coerentemente, é fundamental que $o$ setor agroindustrial desenvolva inovações que favoreçam o sistema de escoamento e armazenamento das safras, reduzindo custos logísticos, tanto para operadores, quanto usuários, possibilitando melhoria no processo produtivo e performance organizacional.

Para Zuurbier (2000) as mudanças tecnológicas sempre influenciaram do modo positivo o sistema agroalimentar, e devido a isso, ocorreram grandes aperfeiçoamentos nos sistemas de suprimento de alimentos devido a inovações na qualidade das sementes, maquinário, nutrição do solo, competências administrativas, sistemas de marketing e assim por diante. $\mathrm{O}$ autor complementa com o 
entendimento de que os produtores assumiram novos papéis na indústria de alimentos mediante a formação de novos arranjos organizacionais, o que lhes possibilitou enfrentar muito melhor as mudanças de mercado.

De acordo com Waack (2000), os processos de gerenciamento da inovação extrapolam os limites das empresas, integrando fornecedores e consumidores em redes globais, e no caso do agronegócio, alguns sistemas industriais toleram menos variações de sofisticação tecnológica. Então o autor, considera que a atenção a este aspecto parece ser fundamental para a formulação das estratégias empresariais.

Não há dúvida que a inovação precisa estar presente no agronegócio, seja na forma de produtos, processos, marketing e organizacional. O Manual de Oslo (2005), considera que as inovações no setor agrícola têm alto impacto econômico, em virtude do elevado peso do setor na economia. Complementa ainda, que em relação ao futuro, torna-se necessário adaptar a metodologia de mensuração da inovação no setor primário, particularmente na agricultura. Consoante isso, o agronegócio brasileiro se destaca por sua eficiência produtiva, porém, a manutenção deste sucesso depende de avanços em novas tecnologias para melhorias na produtividade ou agregando valor ao produto (CICOLIN; OLIVEIRA, 2016; SABADIN, 2016).

No que tange as implicações gerenciais, os achados da pesquisa permitem aos profissionais das áreas afins um melhor entendimento dos benefícios da inovação aplicado a gestão organizacional. O que estimula em estudo futuros a realização de pesquisas empíricas que relacionem a inovação e $o$ agronegócio, comparando diferentes organizações, setores, cadeias produtivas, bem com a percepção dos atores inseridos neste contexto.

\section{REFERÊNCIAS}

ASAI, G. A.; MONTEIRO, M. A.; RODRIGUES, E. A. G.; ROCHA JR., W. F. Risco e governança nas empresas do agronegócio listadas na BMF\&BOVESPA. In: CONGRESSO DA SOCIEDADE BRASILEIRA DE ECONOMIA, ADMINISTRAÇÂO E SOCIOLOGIA RURAL, 55, UFSM, 2017. Anais... Santa Maria: UFSM, 2017.

BANKOLE, F. O.; BANKOLE, O. O. The effects of cultural dimension on ICT innovation: Empirical analysis of mobile phone services. Telematics and Informatics, v. 34, n. 2, p. 490-505, 2017.

BARDIN, L. Análise de conteúdo. Lisboa: Edições 70, 2002.

BATALHA, M. O. As cadeias de produção agroindustriais: uma perspectiva para o estudo das inovações tecnológicas. Revista de Administração da Universidade de São Paulo, v. 30, n. 4, 1995.

\section{CEPEA - CENTRO DE ESTUDOS AVANÇADOS EM ECONOMIA APLICADA. \\ PIB do agronegócio e PIB total do Brasil. Disponível em: 〈http://cepea.esalq.usp.br/pib/>. Acesso em 13 set. de 2016}

CHEN, T.; LIN, Y. C. Feasibility Evaluation and Optimization of a Smart Manufacturing System Based on 3D Printing: A Review. International Journal of Intelligent Systems, 2017.

CICOLIN, L. O. M.; OLIVEIRA, A. L. R. Avaliação de desempenho do processo logístico de exportação do milho brasileiro: uma aplicação da análise envoltória de dados (DEA). Journal of Transport Literature, v. 10, n. 3, p. 30-34, 2016.

CRESWELL, J. W. Projeto de pesquisa: métodos qualitativo, quantitativo e misto. 2. ed. Porto Alegre: Artmed, 2007.

DIAS, M. F. P.; PEDROZO, E. A.; ANICET, C. N. Desafios e respostas inovadoras sustentáveis da agroindústria arrozeira brasileira. Revista em Agronegócio e Meio Ambiente, v. 4, n. 1, 2010. 
DIMASI, J. A.; HANSEN, R. W.; GRABOWSKI, H G. The price of innovation: new estimates of drug development costs. Journal of Health Economics, v. 22 , n. 2, p. 151-185, 2003.

DRUCKER, P. F. Inovação e espírito empreendedor - Entrepreneurship. 6 ed. São Paulo: Pioneira, 1985.

GREENHALGH, T.; ROBERT, G.; MACFARLANE, F.; BATE, P.; KYRIAKIDOU, O. Diffusion of innovations in service organizations: systematic review and recommendations. Milbank Quarterly, v. 82, n. 4, p. 581-629, 2004.

GIBBS, G. Análise de dados qualitativos: Coleção Pesquisa Qualitativa. Porto Alegre, RS: Artmed, 2009.

GUIMARÃES, J. C. F. A relação entre recursos estratégicos e inovação de produto para a obtenção de vantagem competitiva em empresas da indústria moveleira. Tese (Doutorado em Administração). Programa de Pós-graduação em Administração, Universidade de Caxias do Sul - UCS, Caxias do Sul, 2013.

GUPTA, A.; ARORA, N. Understanding determinants and barriers of mobile shopping adoption using behavioral reasoning theory. Journal of Retailing and Consumer Services, v. 36, p. 1-7, 2017.

HANSEN, M. T.; NOHRIA, N.; TIERNEY, T. What's your strategy for managing knowledge? Harvard Business Review, v. 77, n. 3, p. 196-196, 1999.

HERNÁNDEZ, B. J. S. Graphic design and social networks: Methodological proposal supported by the open innovation and co-creation. Advances in Intelligent Systems and Computing, v. 503, p. 297-302, 2017.

JANK, M. S.; NASSAR, A. M.; TACHINARDI, M. H. Agronegócio e comércio exterior brasileiro. Revista USP, n. 64, p. 14-27, 2005.

JUNG, S. H. The contingent effect of analyst coverage: how does analyst coverage affect innovation and Tobin's Q? Asia-Pacific Journal of Accounting \& Economics, v. 24, n. 1-2, p. 43-67, 2017.

KAPLAN, R. S.; NORTON, D. P. The balanced scorecard: measures that drive performance. Harvard Business Review, v. 83, n. 7, p. 172, 1992.

KING, R. P.; BOEHLJE, M.; COOK, M. L.; SONKA, S. T. Agribusiness economics and management. American Journal of Agricultural Economics, v. 92, n. 2, p. 554-570, 2010.

LEONARD- BARTON, D. Core capabilities and core rigidities: A paradox in managing new product devel- opment. Strategic Management Journal, v. 13, n. S1, p. 111-125, 1992.

MANUAL DE OSLO. The measurement of scientific and technological activities. Proposed guidelines for collecting and interpreting innovation data, 2005.

MOORE, G. C.; BENBASAT, I. Development of an instrument to measure the perceptions of adopting an information technology innovation. Information Systems Research, v. 2, n. 3, p. 192-222, 1991.

PARK, K.; KOH, J. Exploring the relationship between perceived pace of technology change and adoption resistance to convergence products. Computers in Human Behavior, v. 69, p. 142-150, 2017.

SAADATMAND, A.; NEMATOLLAHI, A. R.; SADOOGHI-ALVANDI, S. M. On the estimation of missing values in AR (1) model with exponential innovations. Communications in Statistics-Theory and Methods, v. 46, n. 7, p. 3393-3400, 2017.

SABADIN, R. K.; SEVERO, E. A.; GUIMARAES, J. C. F. Inovação em ergonomia, absenteísmo e risco no levantamento de carga. Desenvolve - Revista de Gestão do Unilasalle, v. 6, p. 31-51, 2017.

SABADIN, R. K. As relações entre inovação em ergonomia, absenteísmo e risco no levantamento de carga. Dissertação (Mestrado em Administração). Programa de Pós-graduação em Administração, Faculdade Meridional - IMED, Passo Fundo, 2016.

SADIQ, S.; INDULSKA, M. Open data: Quality over quantity. International Journal of Information Management, v. 37, n. 3, p. 150-154, 2017.

SANTOS, L. P.; AVELAR, J. M. B.; SHIKIDA, P. F. A.; CARVALHO, M. A. Agronegócio brasileiro no comércio internacional. Revista de Ciências Agrárias, v. 39, n.1, p. 54-69, 2016.

SEVERO, E. A. Inovação e sustentabilidade ambiental nas empresas do arranjo produtivo local metalomecânico automotivo da Serra Gaúcha. Tese (Doutorado em Administração). Programa de Pósgraduação em Administração, Universidade de Caxias do Sul - UCS, Caxias do Sul, 2013.

SEVERO, E. A.; DORION, E. C. H.; GUIMARAES, J. C. F. Innovation and environmental sustainability: analysis in Brazilian metal-mechanic industry. International Journal of Innovation and Sustainable Development, v. 11, p. 230-248, 2017.

SCHUMPETER, J. A. The theory of economic development. 1. ed. Cambridge: Harvard University Press, 1934. 
TAYLOR, S.; TODD, P. A. Understanding information technology usage: A test of competing models. Information Systems Research, v. 6, n. 2, p. 144$176,1995$.

TEECE, D. J. Explicating dynamic capabilities: the nature and microfoundations of (sustainable) enterprise performance. Strategic Management Journal, v. 28, n. 13, p. 1319-1350, 2007.

TEECE, D. J.; PISANO, G.; SHUEN, A. Dynamic capabilities and strategic management. Strategic Management Journal, p. 509-533, 1997.

VENKATESH, V.; MORRIS, M. G.; DAVIS, G. B.; DAVIS, F. D. User acceptance of information technology: Toward a unified view. MIS Quarterly, p. 425-478, 2003.

VERMEIR, K.; KELCHTERMANS, G.; MÄRZ, V. Implementing artifacts: An interactive frame analysis of innovative educational practices. Teaching and Teacher Education, v. 63, p. 116-125, 2017.

WAACK, R. S. Gerenciamento de tecnologia e inovação em sistemas agroindustriais. Economia e gestão de negócios agroalimentares. São Paulo: Pioneira/Pensa/USP, p. 323-47, 2000.

WOLFSWINKEL, J. F.; FURTMUELLER, E.;

WILDEROM, C. P. M. Using grounded theory as a method for rigorously reviewing literature. European Journal of Information Systems, v. 22 n. 1, p. 45-55, 2013.

ZUURBIER, P. Cadeias de suprimento nos mercados internacionais. Economia \& Gestão dos Agronegócios. Ed. Pioneira. São Paulo, 2000.

\begin{abstract}
Alessandro Becker
Mestre em Administração pela Faculdade Meridional (IMED). Graduação em Administração pela Pontifícia Universidade Católica do Rio Grande do Sul (PUC/RS). Professor da Faculdade Meridional (IMED).
\end{abstract}

Eliana Andréa Severo
Doutora em Administração pela Pontifícia
Universidade Católica do Rio Grande do Sul
(PUC/RS), e pela Universidade de Caxias do Sul
(UCS). Professora do PPGA da Universidade
Potiguar (UnP).

\section{Julio Cesar Ferro de Guimarães}

Doutor em Administração pela Pontifícia Universidade Católica do Rio Grande do Sul (PUC/RS), e pela Universidade de Caxias do Sul (UCS). Professor do PPGA da Universidade Potiguar (UnP) 